\title{
Microbial fermentation of polyethylene terephthalate (PET) plastic waste for the production of chemicals and electricity
}

\author{
Shafeer Kalathil ${ }^{1,2}$, Melanie Miller ${ }^{1}$, Erwin Reisner ${ }^{1 *}$ \\ ${ }^{1}$ Yusuf Hamied Department of Chemistry, University of Cambridge, Cambridge CB2 1EW, \\ United Kingdom. \\ ${ }^{2} \mathrm{Hub}$ for Biotechnology in the Built Environment, Faculty of Health and Life Sciences, \\ Department of Applied Sciences, Northumbria University, Newcastle, NE1 8ST, United \\ Kingdom
}

*Corresponding author. Email: reisner@ch.cam.ac.uk

\begin{abstract}
Ideonella sakaiensis (I. sakaiensis) can grow on polyethylene terephthalate (PET) as the sole carbon and energy source. Previous work has shown that conversion of the hydrolysis products terephthalic acid (TPA) and ethylene glycol (EG) under aerobic conditions released carbon dioxide and water while yielding adenosine triphosphate (ATP) through oxidative phosphorylation. This study demonstrates that under anaerobic conditions $I$. sakaiensis ferments PET to the feedstock chemicals acetate and ethanol while co-producing ATP by substrate-level phosphorylation. In addition to PET, maltose, EG, and ethanol can also serve as fermenting substrates. Co-culturing of I. sakaiensis with electrogenic Geobacter sulfurreducens produced electricity from PET or EG. This newly identified plastic fermentation process by I. sakaiensis provides a novel biosynthetic route to produce high-value chemicals and electricity from plastic waste streams.
\end{abstract}

Main Text: Synthetic organic polymers known as plastics are commonly used in many applications such as construction, electronics industry, and packaging due to their high durability, low price, easy processability, and low weight, but the majority are discarded after a single use, causing severe environmental concerns (1-3). Among them, polyethylene terephthalate (PET) is widely used in drinking bottles, packaging materials, and fibers in the textile industry. The annual total plastic production in 2019 was 368 million tons of which PETbased plastics contributed approx. 30 million tons (4). Mechanical recycling of PET plastic is widely used but the process results in a decrease in quality with each cycle and the low demand for such lower-quality plastics limits mechanical recycling to a few cycles (5-7). Chemical recycling of PET waste employs hydrolysis, methanolysis, glycolysis, and aminolysis, but these methods usually require high temperature and cause environmental pollution ( 8 ). The nonrecycled PET plastics are mostly disposed by landfilling or incineration (9). Another route for plastics degradation is destruction of larger plastics to micro- and nano-plastics by UV light exposure together with mechanical disruption (10). However, micro- and nano-plastics particles are believed to enter the food chain, which causes a serious concern to health $(11,12)$. Thus, a sustainable and green method to mitigate plastic wastes is highly warranted.

Most of the synthetic plastics, including PET, are chemically inert. Nevertheless, several enzymes and microbes have been identified to break down PET into its corresponding monomers $(10,13)$. Among those enzymes, PETase, carboxylesterase, polyester hydrolase, lipase, and 
cutinase are known for the degradation of PET at ambient temperature and benign $\mathrm{pH}(8,10,14-$ 18). Microbial degradation is slow, but biological methods may ultimately provide a sustainable solution to degrade plastic waste $(10,13,19-21)$. Recently, the bacterium I. sakaiensis has been identified to degrade and assimilate PET as its sole carbon and energy source under aerobic conditions $(13,19-21)$. The bacterium initially adheres on the surface of PET and produces the intermediate mono(2-hydroxyethyl)-terephthalic acid (MHET) by secreting extracellular PETase hydrolases. The MHET is then transported into the periplasm through an outer membrane protein such as porin. MHETase, an intracellular lipoprotein, then hydrolyzes the MHET to TPA and EG. I. sakaiensis metabolizes the hydrolyzed products to yield ATP by oxidative phosphorylation via the tricarboxylic acid (TCA) cycle, but only releases the greenhouse gas $\mathrm{CO}_{2}$ and $\mathrm{H}_{2} \mathrm{O}(19)$.

In this study, we show that I. sakaiensis can ferment PET into acetate and ethanol under anaerobic conditions yielding ATP by substrate-level phosphorylation (Figure 1). In addition to PET, other substrates such as maltose, EG, and ethanol can also be fermented into value-added chemicals by I. sakaiensis. Co-culturing of I. sakaiensis with the bacterium Geobacter sulfurreducens (G. sulfurreducens) attached on a porous inverse opal-indium tin oxide (IO-ITO) electrode in a microbial fuel cell produced electricity from PET or EG, where I. sakaiensis provided acetate as a substrate for G. sulfurreducens from the fermentation of PET or EG. Instead of aerobically producing $\mathrm{CO}_{2}$ and $\mathrm{H}_{2} \mathrm{O}$ as the end products, we demonstrate in this work the anaerobic ability of I. sakaiensis to convert PET into high-value chemicals and, upon coculturing with $G$. sulfurreducens, the generation of electricity. Both pathways provide an economic opportunity to mitigate plastic pollution while co-producing chemicals and energy.

PET and EG fermentation by I. sakaiensis: I. sakaiensis is a gram-negative, rod-shaped bacterium with a cream color (Figure S1) after incubation in the culture medium (NBRC no. 802, Table S1) under aerobic conditions for 24 h. I. sakaiensis is so far considered as an aerobe and was originally isolated from sediments nearby a plastic recycling plant $(19,21)$. Sediments can show frequent shifts between aerobic and anaerobic states (22). Therefore, microbial communities in sediments often show a facultative trait, which means that they can survive in more than one specific condition and do not only show aerobic respiration but also have the ability to ferment and utilize hydrogen. This is often indicated by a rich abundance of fermenting and hydrogenase enzymes in the genome (22). Fermentation usually yields acids and hydrogenases keep the redox balance in fermenting bacteria by regulating the proton-hydrogen equilibrium (23). Therefore, the ability to metabolize hydrogen is frequently observed in anaerobes (24). Specifically, the genome of I. sakaiensis shows the presence of [NiFe] hydrogenases and many fermenting enzymes such as carbon monoxide dehydrogenases, lactate dehydrogenases, alcohol dehydrogenases, acetate kinase among others (Table S2). Additionally, genes predicted to code for proteins involved in the anaerobic metabolism such as iron-sulfur proteins, cytochrome c family proteins, nitrate reductase, nitrite reductase, sulfite reductase, and dimethyl sulfoxide reductases are present in the genome of I. sakaiensis as well as anaerobic regulatory proteins $\mathrm{CRP} / \mathrm{FNR}$ family transcriptional regulator and ferric uptake regulator protein (Fur) (Table S2). The gene analysis therefore reveals a richness of fermenting and anaerobic enzymes in the genome of I. sakaiensis, which inspired us to investigate its fermentative metabolism. 


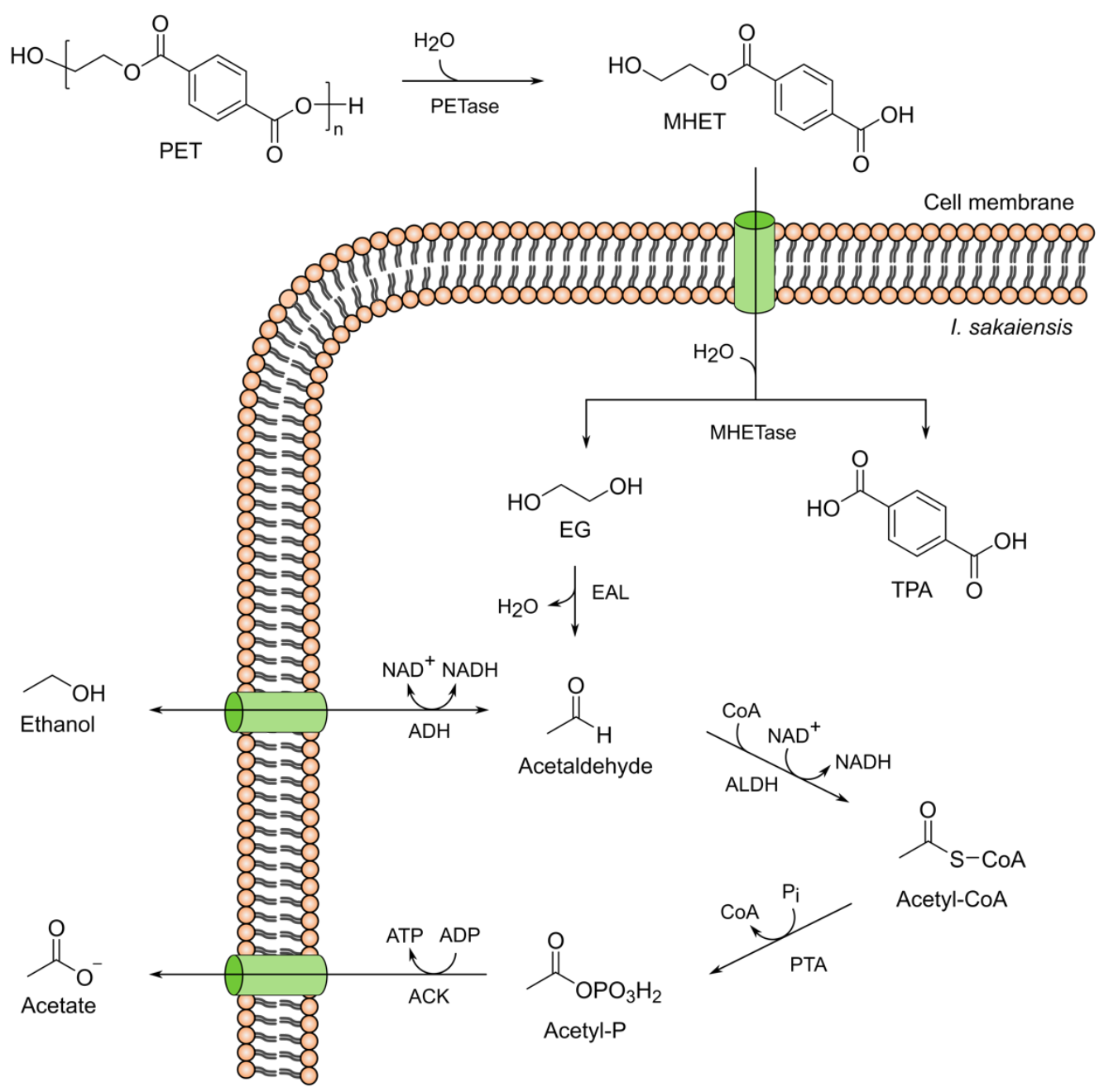

Figure 1. Anaerobic PET conversion by I. sakaiensis. I. sakaiensis secretes the enzyme PETase which converts PET into MHET, which is then transported inside I. sakaiensis and hydrolyzed by MHETase to terephthalic acid (TPA) and ethylene glycol (EG). Under anaerobic conditions, we propose that EG is first dehydrated to acetaldehyde by ethanolamine ammonia lyase (EAL) and then disproportionated by alcohol dehydrogenase (ADH) and aldehyde dehydrogenase (ALDH) to ethanol and acetyl-CoA, respectively. The latter is further converted into acetate by phosphate acetyl transferase (PTA) and acetate kinase (ACK) coupled to ATP formation by substrate-level phosphorylation. Both, acetate and ethanol are transported outside of the cell. Ethanol can reenter the cell and be converted further into acetate (25).

I. sakaiensis can grow aerobically with $\mathrm{PET}$ as the substrate yielding $\mathrm{CO}_{2}$ as the end product (19), and we demonstrate here the fermentative growth of I. sakaiensis with PET as the sole carbon and energy source. The fermentation was carried out with $I$. sakaiensis $\left(\mathrm{OD}_{600}=0.6\right)$ in $15 \mathrm{~mL}$ bicarbonate-buffered complex medium (Table S3) in an anaerobic vial with PET films $(60 \mathrm{mg})$ as the sole carbon source inoculated at ambient conditions $\left(\mathrm{pH} \mathrm{7,30}{ }^{0} \mathrm{C}\right) .{ }^{1} \mathrm{H}$ nuclear magnetic resonance $\left({ }^{1} \mathrm{H}\right.$ NMR) spectroscopy using trimethylsilylpropanoic acid (TSP) as the internal standard for the quantification of the product pool revealed significant acetate production from PET after $5 \mathrm{~d}$ and ethanol production after $15 \mathrm{~d}$ of anaerobic incubation (Figure $2 \mathrm{~A}$ ). No other products have been identified via NMR spectroscopy (Figure S2). This result suggests 
hydrolysis of PET into its monomers (TPA and EG), followed by fermentation of EG into acetate and ethanol. To verify this hypothesis, I. sakaiensis was directly grown on EG as the sole substrate and shown to ferment EG into acetate and ethanol (Figure 2B and S3). Control experiments without $I$. sakaiensis and with heat-killed I. sakaiensis cells did not yield any products (Table S4). Experiments in the absence of PET and EG resulted in no ethanol and minor acetate production after $30 \mathrm{~d}$, which corresponds to less than $2 \%$ of total acetate produced with EG after $22 \mathrm{~d}$ (Table S4). This formation of acetate in the absence of substrate is likely a result of carbon storage compounds inside I. sakaiensis. These control experiments confirm that both, live I. sakaiensis cells and the substrate PET or EG are required for the anaerobic conversion of PET or EG to acetate and ethanol (Table S4).

${ }^{1} \mathrm{H}$ and ${ }^{13} \mathrm{C}$ NMR spectroscopy of experiments with ${ }^{13} \mathrm{C}$ labelled EG as the substrate (Figure $2 \mathrm{C}, \mathrm{D})$ and comparison with commercially available ${ }^{13} \mathrm{C} \mathrm{EG},{ }^{13} \mathrm{C}$ ethanol, and ${ }^{13} \mathrm{C}$ acetate samples (Figure S4, 5) confirmed that the products are derived from EG. ${ }^{12} \mathrm{C}$ acetate peak is also observed, which is the result of the use of internal carbon storage compounds before consumption of the ${ }^{13} \mathrm{C}$ labelled substrate $\mathrm{EG}$.

Based on the NMR spectroscopy data and genome analysis, we propose that under anaerobic conditions, EG is first dehydrated to acetaldehyde by ethanolamine ammonia lyase (EAL) and then disproportionated by alcohol dehydrogenase (ADH) and aldehyde dehydrogenase (ALDH) to ethanol and acetyl-CoA, respectively. The latter is further converted into acetate by phosphate acetyl transferase (PTA) and acetate kinase (ACK) coupled to ATP formation by substrate-level phosphorylation (Figure 1). Both, acetate, and ethanol are transported outside of the cell. Ethanol can reenter the cell and be converted further into acetate (Figure 1). A similar anaerobic EG metabolism was observed in the acetogen Acetobacterium woodii (25). Genome analysis of I. sakaiensis validates our proposed EG metabolism under anaerobic conditions (Figure 1) as all mentioned enzymes (EAL, ADH, ALDH, PTA, and ACK) are present in the bacterial genome (Table S2) (19), except diol dehydratase ( $p d u$ gene) that is commonly employed for dehydration of EG to acetaldehyde (25). However, its homolog EAL (eut gene) is available and previous studies have demonstrated that the eut bacterial microcompartment shares similar features with that of $p d u$ microcompartment in terms of encoded enzymes and chemical reactions (26-29). Further, it has been shown that Salmonella enterica can dehydrate diol to acetaldehyde when a $p d u$ enzyme was replaced by the eut homolog (30). Previous observations therefore confirm that eut gene in I. sakaiensis can dehydrate EG to acetaldehyde.

Ethanol fermentation: We noticed that during EG metabolism, ethanol was further degraded into acetate when EG was almost consumed (Figure 2B). To investigate the possibility of ethanol conversion, we cultured I. sakaiensis directly with ${ }^{13} \mathrm{C}$ labelled ethanol and ${ }^{1} \mathrm{H}$ and ${ }^{13} \mathrm{C}$ NMR spectroscopy showed indeed the gradual fermentation of ethanol to acetate (Figure S6, 7). Based on this observation, we propose that the initially produced ethanol during EG and PET fermentation can reenter the cell, followed by conversion to acetaldehyde by ADH and further fermentation to acetate (Figure 1). 
A

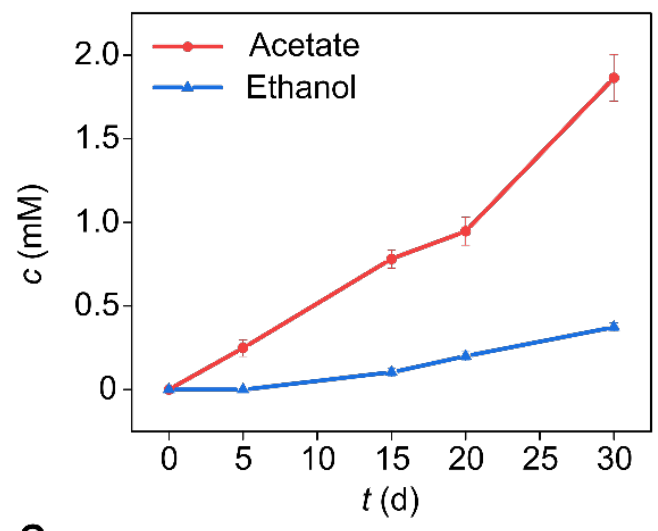

B

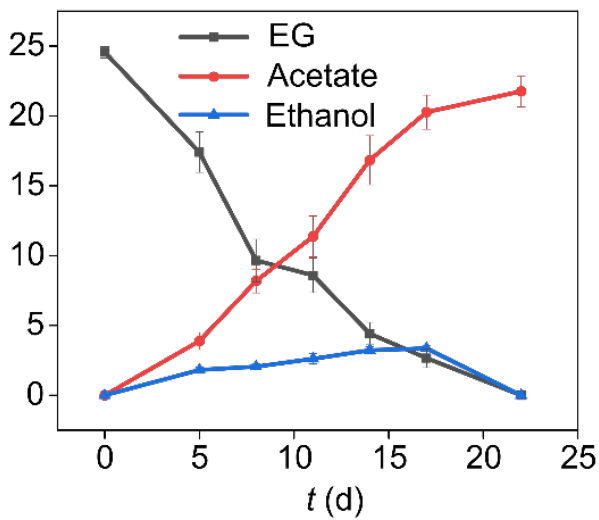

C

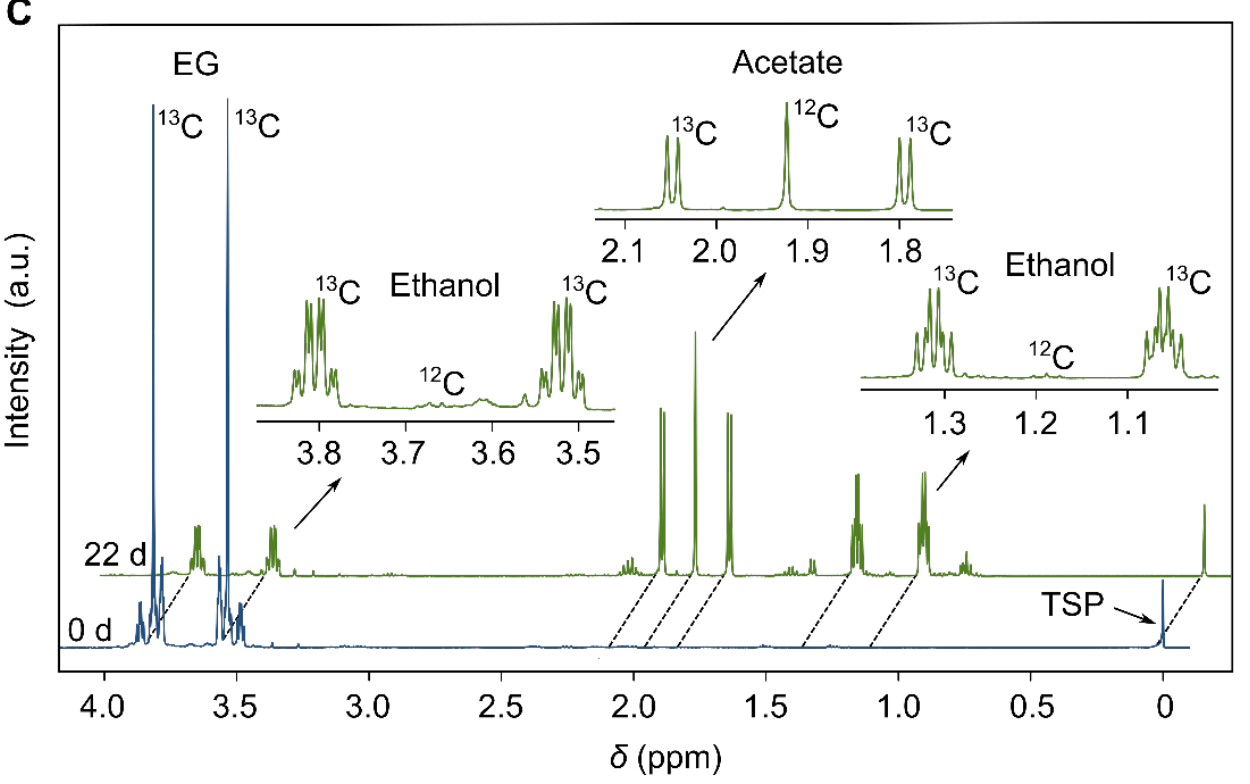

Figure 2. Anaerobic PET and EG fermentation by I. sakaiensis. Conditions: (A) $60 \mathrm{mg}$ PET film and (B) $25 \mathrm{mM}$ EG, I. sakaiensis $\mathrm{OD}_{600}=0.6,15 \mathrm{~mL}$ bicarbonate-buffered complex medium, $\mathrm{N}_{2}-\mathrm{CO}_{2}\left(80-20 \%\right.$ ), shaking incubator, $300 \mathrm{rpm}, 30{ }^{\circ} \mathrm{C}, \mathrm{pH} 7$. Error bars correspond to standard deviation $(\mathrm{N}=3)$. (C) ${ }^{1} \mathrm{H}$ NMR and (D) ${ }^{13} \mathrm{C}$ NMR spectra showing product formation after $22 \mathrm{~d}$ (green) from $25 \mathrm{mM}{ }^{13} \mathrm{C}$ labelled $\mathrm{EG}$ at $0 \mathrm{~d}$ (blue). The TSP signal indicates $0 \mathrm{ppm}$ in each spectrum. The $22 \mathrm{~d}$ spectra are vertically and horizontally shifted as indicated by the black dashed lines. 
Maltose fermentation: It has previously been shown that I. sakaiensis can neither grow aerobically on glucose nor ferment it. The lack of transporters for glucose was found to prevent I. sakaiensis from glucose uptake $(19,21)$. However, aerobic growth with maltose has been verified previously $(19,21)$. Our genome search suggested the possibility of maltose fermentation by I. sakaiensis (Table S2) and fermentative growth of I. sakaiensis was therefore also studied with maltose $(40 \mathrm{mM})$ as the sole carbon source under anaerobic condition. We detected the following fermentation products by ${ }^{1} \mathrm{H}$ NMR spectroscopy: lactate, formate, acetate, and ethanol (Figure S8, 9). Under aerobic condition, maltose is oxidized to $\mathrm{CO}_{2}$ and $\mathrm{H}_{2} \mathrm{O}$ through the TCA cycle (Figure S10). Under anaerobic conditions, maltose is fermented to a product pool via a protein network starting from the outer membrane to the cytoplasm in I. sakaiensis (Figure S10) (31-33). All the necessary proteins are available in the genome of I. sakaiensis with the exception of pyruvate formate lyase (PFL), which has yet to be identified (Table S2) (19).

\section{Co-culturing of I. sakaiensis and G. sulfurreducens in a bio-electrochemical cell: Bacteria} can live in symbiosis, both in natural communities and artificial co-culture systems. The coculturing promotes substrate utilization by serving the metabolites of one community to the neighboring community for their growth $(34,35)$. Here we developed an artificial co-culture system using G. sulfurreducens and I. sakaiensis. G. sulfurreducens usually live with fermenting communities as they grow with acetate, one of the end products of fermentation.

G. sulfurreducens is a gram-negative, anaerobic, dissimilatory metal reducing bacterium with the highest electricity producing capacity in microbial fuel cells. G. sulfurreducens can transport metabolically generated electrons via acetate oxidation to a poised electrode through an extracellular electron transfer (EET) respiratory pathway (36). They also produce conductive protein nanowires for transporting metabolically generated electrons to insoluble electron acceptors such as metal oxide and metal electrodes (37).

We co-cultured I. sakaiensis with G. sulfurreducens in a bio-electrochemical reactor, where I. sakaiensis supplies acetate from fermentation of PET or EG to G. sulfurreducens for electricity production (Figure 3A). The bio-electrochemical reactor consisted of a three-electrode system with a $\mathrm{Ag} / \mathrm{AgCl}$ reference electrode, a Pt mesh counter electrode, and an IO-ITO working electrode (Figure 3B). The IO-ITO electrode served as a host structure for the G. sulfurreducens biofilm and had a geometrical surface area of $0.25 \mathrm{~cm}^{2}$, a thickness of $40-45 \mu \mathrm{m}$, and a macropore size of 8-10 $\mu \mathrm{m}(38,39)$. In the first step towards establishing the artificial co-culture, we grew an electrochemically active $G$. sulfurreducens biofilm on the IO-ITO electrode (IOITO $\mid$ G. sulfurreducens) following a previously published procedure (39) using G. sulfurreducens as the inoculum $\left(\mathrm{OD}_{600}=0.6\right)$ and acetate $(20 \mathrm{mM})$ as the sole carbon source in bicarbonatebuffered complex medium by poising an applied potential of $0.1 \mathrm{~V} v s$. standard hydrogen electrode (SHE). A current plateau at $1.7 \mathrm{~mA} \mathrm{~cm}^{-2}$ was observed after $3 \mathrm{~d}$ (Figure S11), indicating that G. sulfurreducens has colonized on the electrode while metabolizing acetate to $\mathrm{CO}_{2}$ (39). In the second step, the bicarbonate-buffered complex medium was replenished with fresh medium (without acetate and planktonic G. sulfurreducens) and then I. sakaiensis was added $\left(\mathrm{OD}_{600}=0.6\right)$ together with PET films $(60 \mathrm{mg})$ or EG $(25 \mathrm{mM})$ as the sole substrate to the bio-electrochemical reactor containing the IO-ITO $\mid$ G. sulfurreducens electrode. 

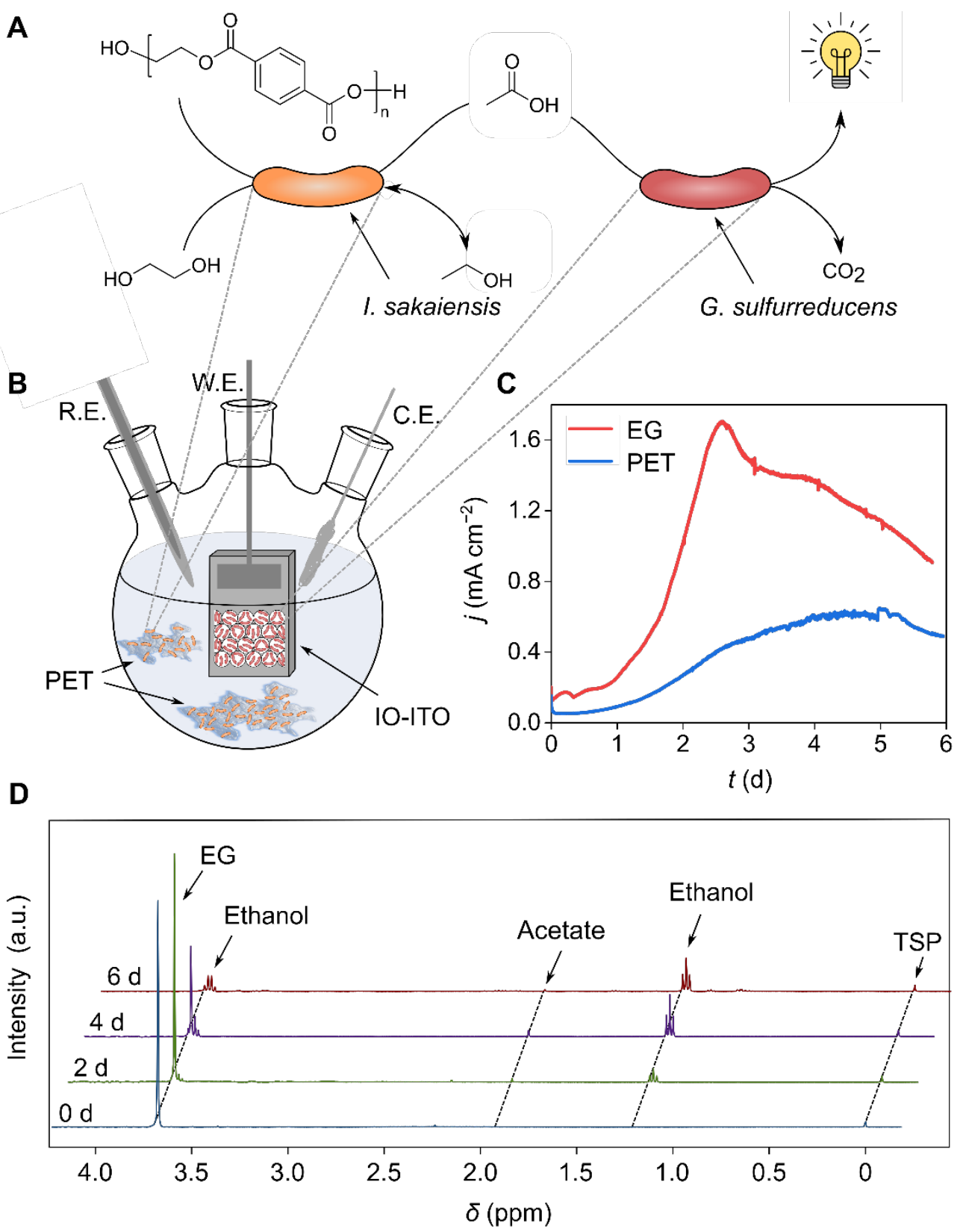

Figure 3. Electricity generation from PET and EG by a co-culture of $I$. sakaiensis and G. sulfurreducens in a three-electrode bio-electrochemical system. (A) PET and EG conversion to electricity and $\mathrm{CO}_{2}$ via intermediate acetate production by a co-culture of I. sakaiensis and G. sulfurreducens. (B) Schematic representation of the co-culturing experiment in a bio-electrochemical cell with a three-electrode system. (C) Conditions: I. sakaiensis OD $_{600}=$ 0.6, IO-ITO $\mid$ G. sulfurreducens working electrode (W.E.) (Figure S11), $60 \mathrm{mg}$ PET (blue) and $25 \mathrm{mM}$ EG (red), $15 \mathrm{~mL}$ bicarbonate-buffered complex medium, $\mathrm{N}_{2}-\mathrm{CO}_{2}(80-20 \%), 30{ }^{\circ} \mathrm{C}$, $400 \mathrm{rpm}, \mathrm{pH}$ 7, $0.10 \mathrm{~V}$ vs. SHE, Ag/AgCl reference electrode (R.E.), Pt mesh counter electrode (C.E.). (D) ${ }^{1} \mathrm{H}$ NMR spectra before the start, after $2 \mathrm{~d}, 4 \mathrm{~d}$, and $6 \mathrm{~d}$ of the chronoamperometry with $25 \mathrm{mM}$ EG. The TSP signal indicates $0 \mathrm{ppm}$ in each spectrum. The spectra are vertically and horizontally shifted as indicated by the black dashed lines. 
The bio-electrochemical system with PET as the sole substrate showed a maximum current density of $0.6 \mathrm{~mA} \mathrm{~cm}{ }^{-2}$ while $\mathrm{EG}$ as the substrate showed a current density of $1.7 \mathrm{~mA} \mathrm{~cm}{ }^{-2}$ (Figure 3C). The lower current from PET compared to EG is attributed to the slower hydrolysis step for the insoluble synthetic polymer. Current production started to decay after an initial peak current for both PET and EG (Figure 3C), which is commonly observed in microbial fuel cells and an indication for a limitation in acetate supply (40).

I. sakaiensis was unable to produce current from EG in the absence of G. sulfurreducens, which implies non-electric behavior of I. sakaiensis (Figure S12). Co-culturing in the absence of PET and EG did not show any electricity production indicating that the oxidation of PET or EG was the source of the observed electricity production (Figure S13). ${ }^{1} \mathrm{H}$ NMR analysis of the EG experiment (Figure 3D) shows a small increase in acetate over time, which reaches $0.8 \mathrm{mM}$ after $4 \mathrm{~d}$ at its highest and then decreases to $0.3 \mathrm{mM}$ after $6 \mathrm{~d}$. For ethanol an increase over time is observed and the concentration is $8.0 \mathrm{mM}$ after $6 \mathrm{~d}$, while EG is completely consumed at this point. The detection of very small amounts of acetate indicates that the acetate produced by I. sakaiensis is instantly consumed by G. sulfurreducens for electricity production. At the same time, ethanol is initially accumulated (Figure 3D) as G. sulfurreducens cannot use ethanol as the carbon source (Figure S14). However, ethanol can be further converted into acetate by I. sakaiensis once EG is fully consumed as observed in the fermentation experiment with the monoculture (Figure 2B). Cross-sectional scanning electron microscopy (SEM) confirmed that bacteria are inside the pores and on top of the IO-ITO electrode (Figure S15).

The experiments show that I. sakaiensis ferments PET or EG to acetate and ethanol while G. sulfurreducens uses the fermented acetate as the substrate for the electricity production while releasing $\mathrm{CO}_{2}$. Overall, the symbiotic system shows a consumption of $16.7 \mathrm{mM}$ EG after $6 \mathrm{~d}$ (Figure 3D) which is converted via acetate into electricity and $\mathrm{CO}_{2}$ and also serves as the carbon source for cell growth of I. sakaiensis and G. sulfurreducens. In contrast, the monoculture only showed a conversion of $9.7 \mathrm{mM}$ after $6 \mathrm{~d}$ (Figure 2B). This 1.7-fold increase in the EG consumption in the co-culture suggests that the metabolism of EG by I. sakaiensis was enhanced when the strain was grown together with $G$. sulfurreducens, where acetate is constantly being consumed. A classic example for the enhanced metabolism in a co-culture system is nitrification. In this syntropic process, ammonium-oxidizing bacteria (AOB) oxidize ammonia to nitrite while nitrite-oxidizing bacteria convert nitrite further to nitrate. This concert operation has been shown to accelerate the performance of $\mathrm{AOB}(34)$.

Conclusion: In conclusion, I. sakaiensis has been known to degrade PET to $\mathrm{CO}_{2}$ under aerobic condition, and we demonstrate here that it can also metabolize PET to acetate and ethanol under anaerobic conditions $\left(30^{\circ} \mathrm{C}, \mathrm{pH} 7,1 \mathrm{~atm}\right)$. This new plastic fermentation process by I. sakaiensis is supported by isotopic labelling studies and genome analysis, which revealed the presence of all the required proteins. The present results confirm that I. sakaiensis is a facultative anaerobe that shows versatile metabolic pathways for PET consumption and utilization under anaerobic and aerobic conditions. Plastic contaminated areas may have a lower oxygen level as plastic materials prevent oxygen penetration from the atmosphere, which could be a reason for the emergence of the observed anaerobic pathway for plastic degradation. This study identifies plastic fermentation as a novel approach to combat plastic pollution and produce high-value chemicals from waste through microbial degradation and biosynthesis. Additionally, the symbiotic association of I. sakaiensis with G. sulfurreducens paves a unique pathway to produce electricity from PET plastic waste. This work therefore reports a novel PET upcycling process, 
termed 'plastic fermentation', which has the potential to emerge as a new technology that combines plastic waste mitigation with the production of value-added chemicals and energy.

\section{References and Notes:}

1. R. Geyer, J. R. Jambeck, K. L. Law, Sci. Adv. 3, 1-5 (2017).

2. S. B. Borrelle, J. Ringma, K. L. Law, C. C. Monnahan, L. Lebreton, A. McGivern, E. Murphy, J. Jambeck, G. H. Leonard, M. A. Hilleary, M. Eriksen, H. P. Possingham, H. De Frond, L. R. Gerber, B. Polidoro, A. Tahir, M. Bernard, N. Mallos, M. Barnes, C. M. Rochman, Science 369, 1515-1518 (2020).

3. J. Brahney, M. Hallerud, E. Heim, M. Hahnenberger, S. Sukumaran, Science 368, 12571260 (2020).

4. "Plastics - the Facts 2020" (Plastics Europe - Association of Plastic Manufacturers, 2020).

5. “Mechanical Recycling” (European Bioplastics e. V., 2020).

6. H. Jeswani, C. Krüger, M. Russ, M. Horlacher, F. Antony, S. Hann, A. Azapagic, Sci. Total Environ. 769, 144483-144498 (2021).

7. Z. O. G. Schyns, M. P. Shaver, Macromol. Rapid Commun. 42, 1-27 (2021).

8. M. Salvador, U. Abdulmutalib, J. Gonzalez, J. Kim, A. A. Smith, J.-L. Faulon, R. Wei, W. Zimmermann, J. I. Jimenez, Genes 10, 373-388 (2019).

9. L. Lebreton, A. Andrady, Palgrave Commun. 5, 6, 1-11 (2019).

10. D. Danso, J. Chow, W. R. Streit, Appl. Environ. Microbiol. 85, 1-14 (2019).

11. X. Lim, Nature 593, 22-25 (2021).

12. R. C. Thompson, C. J. Moore, F. S. vom Saal, S. H. Swan, Philos. Trans. R. Soc. B Biol. Sci. 364, 2153-2166 (2009).

13. U. T. Bornscheuer, Science 351, 1154-1155 (2016).

14. H. P. Austin, M. D. Allen, B. S. Donohoe, N. A. Rorrer, F. L. Kearns, R. L. Silveira, B. C. Pollard, G. Dominick, R. Duman, K. El Omari, V. Mykhaylyk, A. Wagner, W. E. Michener, A. Amore, M. S. Skaf, M. F. Crowley, A. W. Thorne, C. W. Johnson, H. L. Woodcock, J. E. McGeehan, G. T. Beckham, Proc. Natl. Acad. Sci. 115, E4350-E4357 (2018).

15. M. Furukawa, N. Kawakami, A. Tomizawa, K. Miyamoto, Sci. Rep. 9, 16038-16047 (2019).

16. R. Wei, W. Zimmermann, Microb. Biotechnol. 10, 1308-1322 (2017).

17. R. Wei, T. Oeser, J. Schmidt, R. Meier, M. Barth, J. Then, W. Zimmermann, Biotechnol. Bioeng. 113, 1658-1665 (2016).

18. C. M. Carr, D. J. Clarke, A. D. W. Dobson, Front. Microbiol. 11, 1-23 (2020).

19. S. Yoshida, K. Hiraga, T. Takehana, I. Taniguchi, H. Yamaji, Y. Maeda, K. Toyohara, K. Miyamoto, Y. Kimura, K. Oda, Science 351, 1196-1199 (2016). 
20. I. Taniguchi, S. Yoshida, K. Hiraga, K. Miyamoto, Y. Kimura, K. Oda, ACS Catal. 9, 4089-4105 (2019).

21. S. Tanasupawat, T. Takehana, S. Yoshida, K. Hiraga, K. Oda, Int. J. Syst. Evol. Microbiol. 66, 2813-2818 (2016).

22. A. J. Kessler, Y.-J. Chen, D. W. Waite, T. Hutchinson, S. Koh, M. E. Popa, J. Beardall, P. Hugenholtz, P. L. M. Cook, C. Greening, Nat. Microbiol. 4, 1014-1023 (2019).

23. A. Trchounian, R. Gary Sawers, IUBMB Life 66, 1-7 (2014).

24. P. B. Matheus Carnevali, F. Schulz, C. J. Castelle, R. S. Kantor, P. M. Shih, I. Sharon, J. M. Santini, M. R. Olm, Y. Amano, B. C. Thomas, K. Anantharaman, D. Burstein, E. D. Becraft, R. Stepanauskas, T. Woyke, J. F. Banfield, Nat. Commun. 10, 1-15 (2019).

25. D. Trifunović, K. Schuchmann, V. Müller, J. Bacteriol. 198, 1058-1065 (2016).

26. T. O. Yeates, C. S. Crowley, S. Tanaka, Annu. Rev. Biophys. 39, 185-205 (2010).

27. T. Toraya, Chem. Rev. 103, 2095-2128 (2003).

28. M. Semialjac, H. Schwarz, J. Am. Chem. Soc. 124, 8974-8983 (2002).

29. O. Tsoy, D. Ravcheev, A. Mushegian, J. Bacteriol. 191, 7157-7164 (2009).

30. C. M. Jakobson, E. Y. Kim, M. F. Slininger, A. Chien, D. Tullman-Ercek, J. Biol. Chem. 290, 24519-24533 (2015).

31. W. Boos, H. Shuman, Microbiol. Mol. Biol. Rev. 62, 204-229 (1998).

32. S. C. Weiss, A. Skerra, A. Schiefner, J. Biol. Chem. 290, 21352-21364 (2015).

33. R. Dippel, W. Boos, J. Bacteriol. 187, 8322-8331 (2005).

34. R. Tsoi, F. Wu, C. Zhang, S. Bewick, D. Karig, L. You, Proc. Natl. Acad. Sci. 115, 2526-2531 (2018).

35. B. E. L. Morris, R. Henneberger, H. Huber, C. Moissl-Eichinger, FEMS Microbiol. Rev. 37, 384-406 (2013).

36. B. E. Logan, R. Rossi, A. Ragab, P. E. Saikaly, Nat. Rev. Microbiol. 17, 307-319 (2019).

37. D. R. Lovley, J. Yao, Trends Biotechnol. 39, 940-952 (2021).

38. J. Z. Zhang, P. Bombelli, K. P. Sokol, A. Fantuzzi, A. W. Rutherford, C. J. Howe, E. Reisner, J. Am. Chem. Soc. 140, 6-9 (2018).

39. X. Fang, S. Kalathil, G. Divitini, Q. Wang, E. Reisner, Proc. Natl. Acad. Sci. 117, 50745080 (2020).

40. G. Reguera, K. P. Nevin, J. S. Nicoll, S. F. Covalla, T. L. Woodard, D. R. Lovley, Appl. Environ. Microbiol. 72, 7345-7348 (2006).

Acknowledgments: We thank Dr Lin Su and Subhajit Bhattacharjee for proof-reading of the manuscript.

Funding Sources: This work was supported by a European Research Council (ERC) Consolidator Grant 'MatEnSAP' (682833, ER and MM), European Marie Sklodowska-Curie 
individual Fellowships (SK, GAN 744317), UKRI Cambridge Circular Plastics Centre (CirPlas, EP/S025308/1, SK and ER), Funds for Women Graduates Foundation Main Grant (MM), Research England's Expanding Excellence in England (E3) Fund (SK).

Author contributions: SK, MM and ER conceived the idea and designed the project. SK cultured bacteria, performed fermentation, isotopic experiments, coculturing and electricity production from plastics. SK and MM performed NMR analyses. MM recorded SEM images of electrodes and biofilms. SK, MM and ER analyzed the data and discussed the results. SK, MM and ER wrote the manuscript. ER supervised the project.

Competing interests: Authors declare that they have no competing interests.

Data and materials availability: The raw data that support the findings of this study will become available from the University of Cambridge data repository upon acceptance of the manuscript in its final form [DOI will be inserted here before final publication]. 